\title{
Research Designs in Interventional Pain Management: Is Randomization Superior, Desirable or Essential?
}

\author{
Laxmaiah Manchikanti, MD* and Vidyasagar Pampati, MSc ${ }^{\star *}$
}

In the hierarchy of research designs, the results of randomized, controlled trials are considered to be evidence of the highest grade, whereas observational studies are viewed as having less validity because they reportedly overestimate treatment effects. This hierarchy approach to study design has been promoted widely in modern medical literature; in spite of overwhelming evidence that evidence-based medicine includes all types of evidence, and randomized, double-blind studies should not necessarily be considered to represent the best available evidence. In fact, randomized, double-blind studies face insurmountable challenges in interventional pain management. The value of the so-called gold standard of randomized, double-blind trials has been questioned.

This study was undertaken to evaluate if randomization does provide the protective statistical shield that some think it provides in an interventional pain management population. In this study we compared randomized and non-random-

The acme of clinical research is the randomized, doubleblind, controlled trials, but such trials must be undertaken responsibly and are extremely difficult to conduct in interventional pain management. Randomized, controlled trials were introduced into clinical medicine when streptomycin was evaluated in the treatment of tuberculosis (1). Since then, randomized, controlled trials have become the gold standard for assessing the effectiveness of therapeutic agents (2-4). Sacks et al (5) compared published randomized, controlled studies with those that used observational designs. In this landmark evaluation, they showed that the agent being tested was considered effective in 44 of 56 trials (79\%) in observational studies utilizing historical controls, whereas the agent was considered positive in only 10 of $50(20 \%)$ randomized,

From Pain Management Center of Paducah, Paducah, Kentucky. *Dr. Manchikanti is the medical director and **Mr. Pampati is the statistician at Pain Management Center of Paducah. Address correspondence: Laxmaiah Manchikanti, MD, 2831 Lone Oak Road, Paducah, KY 42001. E-mail: drm@ asipp.org ized samples. Randomization was accomplished by the use of random number tables and random sampling into four groups, three or two groups. Non-randomization was achieved by allocation into various groups by two different means.

The results of this evaluation showed that there was only one significant difference when patients were allocated by means of non-randomization among the groups or compared to the total sample. In contrast, randomization showed significant differences in seven parameters.

The results of this study conclude that in interventional pain management settings, non-randomized sampling is valid.

Keywords: Evidence-based medicine, randomized trial, double-blind trial, controlled trial, internal validity, external validity, interventional pain management

controlled trials. Thus, Sacks et al (5) concluded that bias in patient selection may irretrievably weigh the outcome of historically controlled trials in favor of new therapies in observational studies. Advocates of evidence-based medicine classify studies according to the grades of evidence on the basis of the research design, using internal validity as the criterion for hierarchical rankings. The highest grade is reserved for research involving at least one properly randomized, controlled trial; and the lowest grade is applied to descriptive studies and expert opinion; observational studies, cohort studies and case-controlled studies, falling at intermediate levels $(7,8)$. Thus, this hierarchical approach to study design has been promoted widely in individual reports, meta-analysis, consensus statements and educational materials for practitioners.

Evidence-based medicine has been defined by many with contradictory definitions (9). Evidence-based medicine must include all types of evidence, including clinical experience (10-28). However, in spite of the descriptions of evidence-based medicine as including all types of evidence, presently all the decisions are made based on so-called evidence-based medicine derived from 
randomized, double-blind, controlled trials. Numerous stumbling blocks posing challenges to randomized, double-blind trials in interventional pain management have been described (29-36).

The concept that assignment of the subjects randomly to either experimental or controlled status is the perfect science has also been questioned (37). Randomized trials often not only trade internal validity for external validity, but randomization also makes patient recruitment difficult (37). Randomization literally means selection similar to the tossing of a coin, which essentially ensures that the physician responsible for the assignment is not consciously or unconsciously allocating certain patients to a particular group. Thus, criticism has been advanced against various types of non-randomized allocations, including assigning volunteers to the treatment group and those who do not volunteer to the control group, allocation into groups based on alternate days, alternate numbers or another assigned preformed methodology. Finally, it is believed that randomization ensures that the two groups will differ only by chance. However, it does not guarantee that in practice, the balance will be actually achieved through the randomization.

Considering the numerous difficulties with randomization, including patient recruitment, it is not clear that without the manipulation by one of the investigators, other modes of allocation are unbiased and produce similar results in all groups of patients. The distinction between randomization and other non-randomized types of allocation has not been established. This study was undertaken to evaluate various features of patients, presenting to an interventional pain management setting and to compare these features utilizing various types of allocations, including randomization.

\section{METHODS}

This study was designed to evaluate all of the patients seen in one pain management location by one physician during 2001. There were no inclusion or exclusion criteria. Information with respect to gender, age, height, weight, duration, the mode of onset of pain, history of previous surgical intervention and referral pattern was collected for all patients. Further information included data collection of multiple dimensions of pain, psychological status and management.

Phase I or Non-randomized analysis: This phase included analysis of total sample and allocation into four groups by two different means. Initially an evaluation of the total sample with demographic features, regions involved, psychological status, non-physiological features, and clinical approach with results of their follow-ups was performed.

The first part of analysis consisted of allocation of all the patients into four groups. They were assigned a serial number chronologically from 1 to 372 based on their initial date of visit. Patients were allocated into Group I from number 1 to 93; into Group II from 94 to 186; into Group III from 187 to 279; and into Group IV from 280 to 372.

The second part of analysis consisted of allocation of all the patients into another set of four groups. By their assigned serial number, all the patients were divided into four groups, each corresponding to their assigned number, i.e., 1, 5, 9, 13, etc. - Group I; 2, 6, 10, 14, etc. - Group II; $3,7,11,15$, etc. - Group III, 4, 8, 12, 16 etc.- Group IV.

Phase II or Randomized analysis: All 372 patients were divided into either two groups, three groups or four groups by utilizing random tables and finally analyzing 100 patients, divided into 4 groups, with random sampling. Random sampling included 100 of the 372 patients divided into four groups: All the patients were provided with serial numbers from 1 to 372 , according to the initial visit date. Subsequently, 25 patients were selected randomly from serial numbers 1 to 93 using random tables and grouped into Group I; 25 patients from 94 to 186 were grouped into Group II, 25 patients from 187 to 279 were grouped into Group III, and from 280 to 372 into group IV.

Data were recorded on a database using Microsoft ${ }^{\circledR}$ Access $®$; the SPSS version 9.0 statistical package. This package was used to generate the frequency tables and chi-squared statistic was used to test the significance difference between groups. Fischer's exact test was used wherever expected value was less than five. Student's t test was used to test mean difference between groups, and Analysis of variance (ANOVA) is used when comparing more than two groups. Results were considered statistically significant if the p value was less than 0.05 .

\section{RESULTS}

\section{Phase I Analysis}

Results of Phase I analysis are illustrated in Tables 1 and 2 .

Pain Physician Vol. 5, No. 3, 2002 
The data from the total sample is used as baseline data indicating a spectrum of various features in patients presenting to interventional pain management setting. Further analysis consisted of allocation of these patients into four groups based on the date of their initial visit, or allocation by the assigned numbers. The results are illustrated in Tables 1 and 2 with no differences noted between the groups or compared to the total sample in most categories, except in Group I where the percentage of dropouts was $39 \%$, which was significantly higher

Table 1. Results of non-randomized patient allocation into four groups, by serial number based on initial date of visit

\begin{tabular}{|c|c|c|c|c|c|}
\hline Demographic features & $\begin{array}{c}\text { Total } \\
\text { N=372 }\end{array}$ & $\begin{array}{c}\text { Group I } \\
\mathrm{N}=93\end{array}$ & $\begin{array}{c}\text { Group II } \\
\mathbf{N}=93\end{array}$ & $\begin{array}{c}\text { Group III } \\
\mathbf{N}=93\end{array}$ & $\begin{array}{c}\text { Group IV } \\
\mathbf{N}=93\end{array}$ \\
\hline Male & $40 \%$ & $43 \%$ & $34 \%$ & $41 \%$ & $43 \%$ \\
\hline Female & $60 \%$ & $57 \%$ & $66 \%$ & $59 \%$ & $57 \%$ \\
\hline Age (yrs.) & $47+0.8$ & $47+1.6$ & $47+1.7$ & $47+1.4$ & $48+1.7$ \\
\hline Height (inches) & $67+0.2$ & $67+0.37$ & $67+0.38$ & $67+0.44$ & $68+0.41$ \\
\hline Weight (lbs.) & $180+2.4$ & $179+4.4$ & $178+4.0$ & $177+4.1$ & $188+5.5$ \\
\hline Duration of pain (months) & $102+6.4$ & $94+11.5$ & $97+13.5$ & $101+11.4$ & $114+14.6$ \\
\hline Gradual onset & $51 \%$ & $58 \%$ & $51 \%$ & $54 \%$ & $43 \%$ \\
\hline History of surgery & $29 \%$ & $29 \%$ & $32 \%$ & $27 \%$ & $29 \%$ \\
\hline Physician- referral & $69 \%$ & $66 \%$ & $67 \%$ & $73 \%$ & $69 \%$ \\
\hline \multicolumn{6}{|l|}{ Spinal pain } \\
\hline Cervical & $47 \%$ & $40 \%$ & $47 \%$ & $49 \%$ & $53 \%$ \\
\hline Thoracic & $22 \%$ & $23 \%$ & $22 \%$ & $25 \%$ & $19 \%$ \\
\hline Lumbar & $79 \%$ & $75 \%$ & $82 \%$ & $80 \%$ & $81 \%$ \\
\hline Total & $93 \%$ & $89 \%$ & $93 \%$ & $95 \%$ & $96 \%$ \\
\hline \multicolumn{6}{|l|}{ Non-spinal pain } \\
\hline Headache (non-cervicogenic) & $17 \%$ & $24 \%$ & $14 \%$ & $20 \%$ & $11 \%$ \\
\hline Abdominal / chest wall & $7 \%$ & $11 \%$ & $7 \%$ & $7 \%$ & $3 \%$ \\
\hline RSD & $11 \%$ & $18 \%$ & $13 \%$ & $10 \%$ & $3 \%$ \\
\hline \multicolumn{6}{|l|}{ Number of region involved } \\
\hline One region & $100 \%$ & $100 \%$ & $100 \%$ & $100 \%$ & $100 \%$ \\
\hline Two regions & $56 \%$ & $57 \%$ & $55 \%$ & $57 \%$ & $56 \%$ \\
\hline Three regions or more & $22 \%$ & $26 \%$ & $23 \%$ & $27 \%$ & $12 \%$ \\
\hline \multicolumn{6}{|l|}{ Psychological status } \\
\hline Depression & $\mathbf{5 8 \%}$ & $52 \%$ & $53 \%$ & $66 \%$ & $62 \%$ \\
\hline Generalized anxiety disorder & $54 \%$ & $47 \%$ & $50 \%$ & $63 \%$ & $56 \%$ \\
\hline Somatization disorder & $34 \%$ & $27 \%$ & $25 \%$ & $44 \%$ & $39 \%$ \\
\hline \multicolumn{6}{|l|}{ Non-physiologic features } \\
\hline Non-physiologic symptoms & $15 \%$ & $19 \%$ & $11 \%$ & $16 \%$ & $13 \%$ \\
\hline Non-physiologic signs & $15 \%$ & $17 \%$ & $15 \%$ & $14 \%$ & $15 \%$ \\
\hline Symptom magnification & $24 \%$ & $26 \%$ & $18 \%$ & $33 \%$ & $22 \%$ \\
\hline \multicolumn{6}{|l|}{ Clinical approach } \\
\hline Interventional procedures & $74 \%$ & $73 \%$ & $75 \%$ & $72 \%$ & $74 \%$ \\
\hline Non-interventional approach & $19 \%$ & $19 \%$ & $15 \%$ & $20 \%$ & $23 \%$ \\
\hline Evaluation only & $7 \%$ & $8 \%$ & $10 \%$ & $8 \%$ & $3 \%$ \\
\hline Dropout rate & $25 \%$ & 39\%\# & $30 \%$ & $17 \%$ & $13 \%$ \\
\hline
\end{tabular}

\# Indicates significant difference with total sample 
Table 2. Results of non-randomized patient allocation into four groups by sequential allocation of serial numbers

\begin{tabular}{|c|c|c|c|c|c|}
\hline Demographic features & $\begin{array}{c}\text { Group I } \\
\mathrm{N}=93\end{array}$ & $\begin{array}{c}\text { Group II } \\
\mathbf{N}=93\end{array}$ & $\begin{array}{c}\text { Group III } \\
\mathrm{N}=93\end{array}$ & $\begin{array}{c}\text { Group IV } \\
\mathbf{N}=93\end{array}$ & $\begin{array}{c}\text { Total } \\
\mathbf{N}=372\end{array}$ \\
\hline Male & $39 \%$ & $48 \%$ & $40 \%$ & $34 \%$ & $40 \%$ \\
\hline Female & $61 \%$ & $52 \%$ & $60 \%$ & $66 \%$ & $60 \%$ \\
\hline Age (yrs.) & $47+1.4$ & $47+1.7$ & $46+1.6$ & $48+1.7$ & $47+0.8$ \\
\hline Height (inches) & $67+0.3$ & $67+0.4$ & $67+0.4$ & $66+0.4$ & $67+0.2$ \\
\hline Weight (lbs.) & $185+4.0$ & $184+4.8$ & $178+5.5$ & $173+4.5$ & $180+2.4$ \\
\hline Duration of pain (months) & $84+10.2$ & $100+11.2$ & $105+14.3$ & $118+14.6$ & $102+6.4$ \\
\hline Gradual onset & $45 \%$ & $50 \%$ & $52 \%$ & $59 \%$ & $51 \%$ \\
\hline History of surgery & $36 \%$ & $28 \%$ & $28 \%$ & $26 \%$ & $29 \%$ \\
\hline Physician-referral & $69 \%$ & $68 \%$ & $71 \%$ & $67 \%$ & $69 \%$ \\
\hline \multicolumn{6}{|l|}{ Spinal pain } \\
\hline Cervical & $48 \%$ & $54 \%$ & $45 \%$ & $42 \%$ & $47 \%$ \\
\hline Thoracic & $23 \%$ & $18 \%$ & $27 \%$ & $20 \%$ & $22 \%$ \\
\hline Lumbar & $77 \%$ & $81 \%$ & $83 \%$ & $76 \%$ & $79 \%$ \\
\hline Total & $93 \%$ & $93 \%$ & $96 \%$ & $89 \%$ & $93 \%$ \\
\hline \multicolumn{6}{|l|}{ Non-spinal pain } \\
\hline Headache (non-cervicogenic) & $15 \%$ & $16 \%$ & $16 \%$ & $22 \%$ & $17 \%$ \\
\hline Abdominal / chest wall & $5 \%$ & $7 \%$ & $7 \%$ & $7 \%$ & $7 \%$ \\
\hline RSD & $12 \%$ & $13 \%$ & $7 \%$ & $14 \%$ & $11 \%$ \\
\hline \multicolumn{6}{|l|}{ Number of regionsinvolved } \\
\hline One region & $100 \%$ & $100 \%$ & $100 \%$ & $100 \%$ & $100 \%$ \\
\hline Two regions & $55 \%$ & $63 \%$ & $56 \%$ & $50 \%$ & $56 \%$ \\
\hline Three regions or more & $22 \%$ & $21 \%$ & $20 \%$ & $23 \%$ & $22 \%$ \\
\hline \multicolumn{6}{|l|}{ Psychological status } \\
\hline Depression & $58 \%$ & $63 \%$ & $55 \%$ & $56 \%$ & $\mathbf{5 8 \%}$ \\
\hline Generalized anxiety disorder & $57 \%$ & $50 \%$ & $58 \%$ & $52 \%$ & $54 \%$ \\
\hline Somatization disorder & $33 \%$ & $36 \%$ & $34 \%$ & $31 \%$ & $34 \%$ \\
\hline \multicolumn{6}{|l|}{ Non-physiologic features } \\
\hline Non-physiologic symptoms & $14 \%$ & $16 \%$ & $17 \%$ & $12 \%$ & $15 \%$ \\
\hline Non-physiologic signs & $13 \%$ & $17 \%$ & $17 \%$ & $14 \%$ & $15 \%$ \\
\hline Symptom magnification & $27 \%$ & $26 \%$ & $26 \%$ & $20 \%$ & $24 \%$ \\
\hline \multicolumn{6}{|l|}{ Clinical approach } \\
\hline Interventional procedures & $75 \%$ & $68 \%$ & $73 \%$ & $79 \%$ & $74 \%$ \\
\hline Non-interventional approach & $17 \%$ & $21 \%$ & $22 \%$ & $17 \%$ & $19 \%$ \\
\hline Evaluation only & $8 \%$ & $11 \%$ & $5 \%$ & $4 \%$ & $7 \%$ \\
\hline Dropout rate & $25 \%$ & $28 \%$ & $20 \%$ & $24 \%$ & $25 \%$ \\
\hline
\end{tabular}

compared to total dropouts, which was $25 \%$ (Table 1), when patients were allocated by serial numbers.

\section{Phase II Analysis}

Table 3 shows random numbers utilized in analysis.
Table 4 shows the results of Phase II analysis with randomization into various groups. Significant differences were noted as follows: 1) involvement of cervical region was higher in Group II compared to Group I, when patients were randomized into two groups; 2) incidence of symptom magnification was lower in Group II and III, 
Table 3: Random numbers utilized in randomization

\begin{tabular}{|c|c|c|c|c|c|c|c|c|c|c|c|c|c|c|c|c|c|c|c|c|c|c|c|}
\hline \multicolumn{6}{|c|}{$\begin{array}{c}\text { Randomization into Two } \\
\text { Groups }\end{array}$} & \multicolumn{6}{|c|}{$\begin{array}{c}\text { Randomization into Three } \\
\text { Groups }\end{array}$} & \multicolumn{8}{|c|}{ Randomization into Four Groups } & \multicolumn{4}{|c|}{ Random Sampling } \\
\hline \multicolumn{3}{|c|}{ I } & & II & & & I & I & II & III & & I & & Il & & I & II & IV & & I & II & III & IV \\
\hline 1 & 137 & 262 & 4 & 121 & 236 & 4 & 206 & 2 & 199 & 1 & 158 & 1 & 176 & 7 & 196 & 3 & 214 & 4 & 188 & 5 & 10 & 17 & 3 \\
\hline 2 & 140 & 264 & 7 & 122 & 237 & 8 & 207 & 3 & 202 & 6 & 160 & 2 & 177 & 26 & 201 & 5 & 216 & 8 & 191 & 16 & 12 & 26 & 28 \\
\hline 3 & 142 & 265 & 12 & 123 & 240 & 11 & 211 & 5 & 204 & 14 & 161 & 6 & 180 & 29 & 204 & 9 & 218 & 14 & 197 & 33 & 30 & 35 & 74 \\
\hline 5 & 143 & 267 & 14 & 124 & 241 & 13 & 214 & 7 & 209 & 15 & 165 & 10 & 181 & 38 & 206 & 16 & 219 & 17 & 198 & 37 & 32 & 36 & 90 \\
\hline 6 & 147 & 269 & 16 & 126 & 242 & 16 & 222 & 9 & 210 & 17 & 166 & 11 & 182 & 40 & 210 & 20 & 223 & 18 & 199 & 40 & 34 & 82 & 96 \\
\hline 8 & 148 & 270 & 17 & 127 & 244 & 21 & 224 & 10 & 212 & 19 & 169 & 12 & 183 & 43 & 215 & 23 & 229 & 19 & 205 & 70 & 38 & 88 & 97 \\
\hline 9 & 152 & 271 & 18 & 129 & 246 & 24 & 225 & 12 & 217 & 20 & 171 & 13 & 185 & 52 & 235 & 31 & 230 & 27 & 207 & 95 & 57 & 105 & 115 \\
\hline 10 & 154 & 272 & 20 & 130 & 250 & 25 & 227 & 18 & 218 & 22 & 173 & 15 & 190 & 54 & 237 & 37 & 231 & 36 & 208 & 107 & 60 & 125 & 156 \\
\hline 11 & 155 & 274 & 23 & 131 & 251 & 26 & 229 & 29 & 219 & 23 & 174 & 21 & 202 & 57 & 241 & 39 & 234 & 41 & 217 & 111 & 91 & 138 & 158 \\
\hline 13 & 156 & 276 & 25 & 132 & 252 & 28 & 234 & 30 & 220 & 27 & 177 & 22 & 203 & 59 & 242 & 45 & 236 & 46 & 221 & 112 & 93 & 171 & 172 \\
\hline 15 & 157 & 280 & 26 & 135 & 253 & 32 & 235 & 31 & 228 & 33 & 180 & 24 & 209 & 76 & 243 & 47 & 244 & 48 & 224 & 123 & 94 & 193 & 189 \\
\hline 19 & 158 & 281 & 27 & 136 & 255 & 34 & 239 & 36 & 232 & 35 & 189 & 25 & 211 & 77 & 245 & 50 & 246 & 49 & 225 & 124 & 122 & 198 & 207 \\
\hline 21 & 161 & 284 & 31 & 138 & 261 & 42 & 241 & 37 & 236 & 40 & 191 & 28 & 220 & 79 & 247 & 56 & 248 & 53 & 227 & 128 & 145 & 218 & 211 \\
\hline 22 & 164 & 287 & 36 & 139 & 263 & 52 & 242 & 38 & 237 & 44 & 201 & 30 & 222 & 83 & 249 & 58 & 258 & 55 & 228 & 130 & 147 & 230 & 212 \\
\hline 24 & 167 & 288 & 37 & 141 & 266 & 55 & 243 & 39 & 238 & 45 & 203 & 32 & 226 & 86 & 250 & 62 & 261 & 60 & 232 & 190 & 187 & 235 & 215 \\
\hline 28 & 170 & 289 & 38 & 144 & 268 & 57 & 245 & 41 & 244 & 46 & 205 & 33 & 238 & 91 & 251 & 63 & 263 & 65 & 233 & 199 & 194 & 248 & 233 \\
\hline 29 & 171 & 290 & 41 & 145 & 273 & 58 & 247 & 43 & 248 & 48 & 208 & 34 & 239 & 93 & 252 & 64 & 266 & 70 & 240 & 204 & 195 & 259 & 245 \\
\hline 30 & 173 & 291 & 43 & 146 & 275 & 59 & 249 & 47 & 250 & 49 & 213 & 35 & 254 & 94 & 253 & 68 & 270 & 78 & 255 & 206 & 217 & 271 & 252 \\
\hline 32 & 176 & 293 & 45 & 149 & 277 & 62 & 251 & 50 & 252 & 53 & 215 & 42 & 256 & 96 & 260 & 69 & 278 & 81 & 259 & 209 & 223 & 274 & 278 \\
\hline 33 & 177 & 295 & 46 & 150 & 278 & 67 & 254 & 51 & 253 & 60 & 216 & 44 & 257 & 98 & 269 & 72 & 280 & 84 & 268 & 239 & 229 & 289 & 305 \\
\hline 34 & 180 & 297 & 47 & 151 & 279 & 68 & 256 & 54 & 255 & 61 & 221 & 51 & 262 & 102 & 273 & 75 & 286 & 95 & 272 & 281 & 254 & 300 & 328 \\
\hline 35 & 181 & 298 & 49 & 153 & 282 & 69 & 258 & 56 & 259 & 63 & 223 & 61 & 264 & 104 & 283 & 82 & 291 & 97 & 275 & 298 & 273 & 307 & 329 \\
\hline 39 & 182 & 299 & 50 & 159 & 283 & 72 & 262 & 65 & 260 & 64 & 226 & 66 & 265 & 107 & 285 & 87 & 294 & 101 & 277 & 327 & 290 & 309 & 353 \\
\hline 40 & 183 & 300 & 51 & 160 & 285 & 73 & 263 & 66 & 264 & 70 & 230 & 67 & 267 & 108 & 289 & 88 & 297 & 106 & 279 & 346 & 330 & 315 & 359 \\
\hline 42 & 184 & 302 & 52 & 162 & 286 & 76 & 270 & 74 & 265 & 71 & 231 & 71 & 271 & 110 & 290 & 89 & 305 & 109 & 281 & 357 & 371 & 335 & 369 \\
\hline 44 & 190 & 304 & 53 & 163 & 292 & 77 & 274 & 85 & 267 & 75 & 233 & 73 & 274 & 118 & 301 & 100 & 306 & 112 & 282 & & & & \\
\hline 48 & 191 & 309 & 55 & 165 & 294 & 78 & 277 & 86 & 269 & 79 & 240 & 74 & 276 & 121 & 303 & 111 & 307 & 113 & 284 & & & & \\
\hline 54 & 196 & 310 & 56 & 166 & 296 & 82 & 279 & 92 & 271 & 80 & 246 & 80 & 287 & 123 & 304 & 114 & 317 & 117 & 288 & & & & \\
\hline 57 & 198 & 312 & 59 & 168 & 301 & 83 & 282 & 93 & 272 & 81 & 257 & 85 & 293 & 129 & 308 & 115 & 318 & 126 & 292 & & & & \\
\hline 58 & 200 & 313 & 63 & 169 & 303 & 84 & 283 & 98 & 276 & 87 & 261 & 90 & 295 & 130 & 311 & 116 & 326 & 134 & 296 & & & & \\
\hline 60 & 201 & 316 & 64 & 172 & 305 & 88 & 286 & 99 & 280 & 89 & 266 & 92 & 299 & 131 & 312 & 122 & 328 & 137 & 298 & & & & \\
\hline 61 & 202 & 319 & 65 & 174 & 306 & 90 & 288 & 104 & 281 & 91 & 268 & 99 & 300 & 141 & 313 & 124 & 331 & 138 & 302 & & & & \\
\hline 62 & 203 & 321 & 72 & 175 & 307 & 103 & 289 & 105 & 284 & 94 & 273 & 103 & 309 & 145 & 315 & 125 & 333 & 139 & 310 & & & & \\
\hline 66 & 204 & 323 & 73 & 178 & 308 & 109 & 291 & 106 & 287 & 95 & 275 & 105 & 316 & 146 & 320 & 127 & 334 & 144 & 314 & & & & \\
\hline 67 & 205 & 324 & 76 & 179 & 311 & 113 & 296 & 110 & 290 & 96 & 278 & 119 & 321 & 156 & 322 & 132 & 337 & 149 & 319 & & & & \\
\hline 68 & 208 & 325 & 77 & 185 & 314 & 117 & 297 & 112 & 303 & 97 & 285 & 120 & 323 & 159 & 327 & 135 & 342 & 150 & 325 & & & & \\
\hline 69 & 209 & 329 & 78 & 186 & 315 & 118 & 299 & 114 & 307 & 100 & 292 & 128 & 324 & 162 & 338 & 136 & 351 & 151 & 332 & & & & \\
\hline 70 & 211 & 330 & 79 & 187 & 317 & 119 & 300 & 116 & 308 & 101 & 293 & 133 & 329 & 164 & 341 & 142 & 355 & 153 & 335 & & & & \\
\hline 71 & 212 & 331 & 83 & 188 & 318 & 123 & 301 & 122 & 310 & 102 & 294 & 140 & 330 & 166 & 344 & 143 & 356 & 157 & 336 & & & & \\
\hline 74 & 214 & 334 & 84 & 189 & 320 & 125 & 304 & 130 & 312 & 107 & 295 & 147 & 343 & 170 & 346 & 154 & 359 & 160 & 339 & & & & \\
\hline 75 & 219 & 335 & 87 & 192 & 322 & 127 & 305 & 140 & 313 & 108 & 298 & 148 & 345 & 171 & 357 & 155 & 360 & 165 & 340 & & & & \\
\hline 80 & 220 & 339 & 88 & 193 & 326 & 131 & 311 & 142 & 314 & 111 & 302 & 152 & 348 & 179 & 358 & 167 & 361 & 168 & 347 & & & & \\
\hline 81 & 221 & 342 & 89 & 194 & 327 & 134 & 319 & 146 & 315 & 115 & 306 & 158 & 349 & 184 & 362 & 194 & 365 & 169 & 350 & & & & \\
\hline 82 & 222 & 343 & 91 & 195 & 328 & 135 & 326 & 148 & 316 & 120 & 309 & 161 & 353 & 187 & 363 & 195 & 366 & 172 & 352 & & & & \\
\hline 85 & 224 & 344 & 93 & 197 & 332 & 139 & 331 & 150 & 320 & 121 & 317 & 163 & 364 & 189 & 367 & 205 & 369 & 174 & 354 & & & & \\
\hline 86 & 226 & 345 & 94 & 199 & 333 & 149 & 332 & 157 & 325 & 124 & 318 & 173 & 370 & 192 & 372 & 212 & 371 & 178 & 368 & & & & \\
\hline 90 & 229 & 346 & 96 & 206 & 336 & 152 & 333 & 159 & 328 & 126 & 321 & 175 & & 193 & & 213 & & 186 & & & & & \\
\hline 92 & 233 & 347 & 97 & 207 & 337 & 153 & 336 & 162 & 335 & 128 & 322 & & & & & & & & & & & & \\
\hline 95 & 234 & 348 & 98 & 210 & 338 & 154 & 338 & 164 & 337 & 129 & 323 & & & & & & & & & & & & \\
\hline 99 & 238 & 349 & 101 & 213 & 340 & 163 & 342 & 175 & 340 & 132 & 32 & & & & & & & & & & & & \\
\hline 100 & 239 & 352 & 102 & 215 & 341 & 167 & 343 & 176 & 341 & 133 & 327 & & & & & & & & & & & & \\
\hline 104 & 243 & 353 & 103 & 216 & 350 & 168 & 344 & 178 & 348 & 136 & 329 & & & & & & & & & & & & \\
\hline 105 & 245 & 354 & 106 & 217 & 351 & 170 & 345 & 181 & 350 & 137 & 33 & & & & & & & & & & & & \\
\hline 107 & 247 & 357 & 108 & 218 & 355 & 172 & 347 & 183 & 351 & 138 & 334 & & & & & & & & & & & & \\
\hline 117 & 248 & 362 & 109 & 223 & 356 & 179 & 353 & 186 & 354 & 141 & 339 & & & & & & & & & & & & \\
\hline 118 & 249 & 363 & 110 & 225 & 358 & 182 & 355 & 187 & 356 & 143 & 346 & & & & & & & & & & & & \\
\hline 119 & 254 & 364 & 111 & 227 & 359 & 184 & 359 & 188 & 357 & 144 & 349 & & & & & & & & & & & & \\
\hline 120 & 256 & 366 & 112 & 228 & 360 & 185 & 361 & 190 & 358 & 145 & 352 & & & & & & & & & & & & \\
\hline 125 & 257 & 367 & 113 & 230 & 361 & 193 & 363 & 192 & 360 & 147 & 362 & & & & & & & & & & & & \\
\hline 128 & 258 & 368 & 114 & 231 & 365 & 194 & 367 & 195 & 365 & 151 & 36 & & & & & & & & & & & & \\
\hline 133 & 259 & 370 & 115 & 232 & 369 & 197 & 368 & 196 & 366 & 155 & 37 & & & & & & & & & & & & \\
\hline 134 & 260 & 371 & 116 & 235 & 372 & 205 & 372 & 198 & 369 & 156 & 371 & & & & & & & & & & & & \\
\hline
\end{tabular}


Table 4. Results of patient allocation by randomization and random sampling

\begin{tabular}{|c|c|c|c|c|c|c|c|c|c|c|c|c|c|c|}
\hline \multirow[t]{2}{*}{$\begin{array}{l}\text { Demographic } \\
\text { features }\end{array}$} & \multirow[b]{2}{*}{$\begin{array}{c}\text { Total } \\
\mathbf{N}=\mathbf{3 7 2}\end{array}$} & \multicolumn{2}{|c|}{$\begin{array}{c}\text { Randomization into } \\
\text { Two Groups }\end{array}$} & \multicolumn{3}{|c|}{ Randomization into Three Groups } & \multicolumn{4}{|c|}{ Randomization into Four Groups } & \multicolumn{4}{|c|}{ Random Sampling } \\
\hline & & $\begin{array}{c}\mathrm{I} \\
\mathrm{N}=186\end{array}$ & $\begin{array}{c}\mathrm{II} \\
\mathrm{N}=186\end{array}$ & $\begin{array}{c}\mathrm{I} \\
\mathrm{N}=124\end{array}$ & $\begin{array}{c}\mathrm{II} \\
\mathrm{N}=124\end{array}$ & $\begin{array}{c}\text { III } \\
\mathrm{N}=124\end{array}$ & $\begin{array}{c}\mathrm{I} \\
\mathrm{N}=93\end{array}$ & $\begin{array}{c}\mathrm{II} \\
\mathrm{N}=93\end{array}$ & $\begin{array}{c}\text { III } \\
\mathrm{N}=93\end{array}$ & $\begin{array}{c}\mathrm{IV} \\
\mathrm{N}=93\end{array}$ & $\begin{array}{c}\mathrm{I} \\
\mathrm{N}=25\end{array}$ & $\begin{array}{c}\mathrm{II} \\
\mathrm{N}=25\end{array}$ & $\begin{array}{c}\text { III } \\
\mathrm{N}=25\end{array}$ & $\begin{array}{c}\mathrm{IV} \\
\mathrm{N}=25\end{array}$ \\
\hline Male & $40 \%$ & $42 \%$ & $38 \%$ & $42 \%$ & $36 \%$ & $44 \%$ & $39 \%$ & $42 \%$ & $38 \%$ & $43 \%$ & $36 \%$ & $52 \%$ & $28 \%$ & $32 \%$ \\
\hline Female & $60 \%$ & $58 \%$ & $62 \%$ & $58 \%$ & $65 \%$ & $57 \%$ & $61 \%$ & $58 \%$ & $62 \%$ & $57 \%$ & $64 \%$ & $48 \%$ & $72 \%$ & $68 \%$ \\
\hline Age (yrs.) & $47+0.8$ & $46+1.1$ & $48+1.1$ & $45+1.3$ & $48+1.4$ & $48+1.4$ & $43 \#+1.4$ & $48+1.7$ & $49+1.6$ & $47+1.6$ & $50+3.3$ & $40 \#+2.3$ & $49+3.0$ & $46+2.8$ \\
\hline Height (inches) & $67+0.2$ & $67+0.3$ & $67+0.3$ & $67+0.3$ & $67+0.3$ & $67+0.4$ & $67+0.4$ & $67+0.4$ & $67+0.4$ & $67+0.4$ & $67+0.7$ & $67+0.6$ & $67+0.8$ & $67+0.8$ \\
\hline Weight (lbs.) & $180+2.4$ & $184+3.6$ & $177+3.1$ & $178+4.1$ & $175+3.8$ & $187+4.4$ & $185+4.9$ & $181+4.6$ & $176+4.4$ & $179+5.3$ & $187+9.1$ & $188+10.2$ & $175+9.7$ & $171+7.6$ \\
\hline $\begin{array}{l}\text { Duration of pain } \\
\text { (months) }\end{array}$ & $102+2.4$ & $92+8.6$ & $111+9.5$ & $108+10.7$ & $82+9.5$ & $116+12.6$ & $87+10.9$ & $96+12.6$ & $99+13.0$ & $125+14.3$ & $92+16.9$ & $86+24.2$ & $97+24.1$ & $152+37.6$ \\
\hline Gradual onset & $51 \%$ & $47 \%$ & $55 \%$ & $52 \%$ & $52 \%$ & $51 \%$ & $41 \%$ & $53 \%$ & $59 \% *$ & $53 \%$ & $72 \%$ & $40 \%$ & $48 \%$ & $52 \%$ \\
\hline \multicolumn{15}{|c|}{ Involvement of regions } \\
\hline Cervical & $47 \%$ & $43 \%$ & $53 \% *$ & $44 \%$ & $56 \%$ & $52 \%$ & $47 \%$ & $46 \%$ & $49 \%$ & $46 \%$ & $48 \%$ & $60 \%$ & $52 \%$ & $60 \%$ \\
\hline Thoracic & $22 \%$ & $22 \%$ & $21 \%$ & $24 \%$ & $20 \%$ & $22 \%$ & $25 \%$ & $21 \%$ & $16 \%$ & $26 \%$ & $20 \%$ & $12 \%$ & $20 \%$ & $20 \%$ \\
\hline Lumbar & $79 \%$ & $81 \%$ & $77 \%$ & $82 \%$ & $77 \%$ & $78 \%$ & $79 \%$ & $75 \%$ & $79 \%$ & $85 \%$ & $64 \%$ & $72 \%$ & $84 \%$ & $80 \%$ \\
\hline \multicolumn{15}{|c|}{ Number of regions } \\
\hline One region & $100 \%$ & $100 \%$ & $100 \%$ & $100 \%$ & $100 \%$ & $100 \%$ & $100 \%$ & $100 \%$ & $100 \%$ & $100 \%$ & $56 \%$ & $44 \%$ & $44 \%$ & $48 \%$ \\
\hline Two regions & $56 \%$ & $54 \%$ & $58 \%$ & $52 \%$ & $58 \%$ & $58 \%$ & $57 \%$ & $56 \%$ & $54 \%$ & $58 \%$ & $24 \%$ & $40 \%$ & $32 \%$ & $36 \%$ \\
\hline $\begin{array}{l}\text { Three regions or } \\
\text { more }\end{array}$ & $22 \%$ & $20 \%$ & $23 \%$ & $25 \%$ & $18 \%$ & $23 \%$ & $24 \%$ & $17 \%$ & $17 \%$ & $29 \%$ & $20 \%$ & $16 \%$ & $24 \%$ & $16 \%$ \\
\hline \multicolumn{15}{|c|}{ Psychological status } \\
\hline Depression & $\mathbf{5 8 \%}$ & $61 \%$ & $55 \%$ & $61 \%$ & $57 \%$ & $57 \%$ & $63 \%$ & $53 \%$ & $63 \%$ & $53 \%$ & $60 \%$ & $44 \%$ & $68 \%$ & $72 \%$ \\
\hline $\begin{array}{l}\text { Generalized } \\
\text { anxiety disorder }\end{array}$ & $54 \%$ & $55 \%$ & $53 \%$ & $58 \%$ & $51 \%$ & $53 \%$ & $58 \%$ & $45 \%$ & $60 \%$ & $53 \%$ & $52 \%$ & $44 \%$ & $72 \%$ & $72 \%$ \\
\hline $\begin{array}{l}\text { Somatization } \\
\text { disorder } \\
\text { Non-physiologic }\end{array}$ & $\begin{array}{l}34 \% \\
\text { features }\end{array}$ & $34 \%$ & $33 \%$ & $40 \%$ & $32 \%$ & $30 \%$ & $37 \%$ & $24 \%$ & $41 \%$ & $33 \%$ & $32 \%$ & $20 \%$ & $52 \%$ & $44 \%$ \\
\hline $\begin{array}{l}\text { Non-physiologic } \\
\text { symptoms }\end{array}$ & $15 \%$ & $14 \%$ & $16 \%$ & $21 \%$ & $12 \%$ & $11 \%$ & $14 \%$ & $9 \%$ & $16 \%$ & $20 \%$ & $16 \%$ & $12 \%$ & $16 \%$ & $20 \%$ \\
\hline $\begin{array}{l}\text { Non-physiologic } \\
\text { signs }\end{array}$ & $15 \%$ & $14 \%$ & $16 \%$ & $21 \%$ & $14 \%$ & $11 \%$ & $16 \%$ & $11 \%$ & $17 \%$ & $17 \%$ & $12 \%$ & $8 \%$ & $12 \%$ & $24 \%$ \\
\hline $\begin{array}{l}\text { Symptom } \\
\text { magnification } \\
\text { Clinical approac }\end{array}$ & $24 \%$ & $24 \%$ & $24 \%$ & $34 \%$ & $19 \% *$ & $21 \% *$ & $27 \%$ & $17 \%$ & $29 \%$ & $26 \%$ & $20 \%$ & $16 \%$ & $28 \%$ & $32 \%$ \\
\hline $\begin{array}{l}\text { Interventional } \\
\text { procedures }\end{array}$ & $74 \%$ & $73 \%$ & $74 \%$ & $75 \%$ & $69 \%$ & $77 \%$ & $75 \%$ & $71 \%$ & $68 \%$ & $81 \%$ & $88 \%$ & $64 \%$ & $72 \%$ & $80 \%$ \\
\hline $\begin{array}{l}\text { Non-interventio- } \\
\text { nal approach }\end{array}$ & $19 \%$ & $21 \%$ & $18 \%$ & $19 \%$ & $24 \%$ & $15 \%$ & $22 \%$ & $18 \%$ & $21 \%$ & $16 \%$ & $12 \%$ & $20 \%$ & $8 \%$ & $4 \%$ \\
\hline Evaluation only & $7 \%$ & $6 \%$ & $8 \%$ & $6 \%$ & $7 \%$ & $8 \%$ & $3 \%$ & $11 \%$ & $11 \%$ & $3 \%$ & $0 \%$ & $16 \%$ & $20 \%$ & $16 \%$ \\
\hline Dropout rate & $25 \%$ & $22 \%$ & $26 \%$ & $22 \%$ & $26 \%$ & $26 \%$ & $18 \% \#$ & $27 \%$ & $26 \%$ & $27 \%$ & $44 \% \#$ & $20 \%$ & $12 \%$ & $16 \%$ \\
\hline
\end{tabular}

* Indicates significant difference with Group I

\# Indicates significant difference with Total sample

compared to Group I, with randomization into three groups; 3) mean age was lower in Group I, compared to total sample, with randomization into four groups; 4) incidence of dropouts was lower in Group I, compared to total sample with randomization into four groups; 5) incidence of gradual onset of pain was higher in Group
III, compared to Group I, with randomization into four groups; 6) mean age was lower in Group II, compared to total sample with random sampling; and 7) incidence of dropouts was higher in Group I, compared to total sample with random sampling. 


\section{DISCUSSION}

Systematic reviews and meta-analysis offer an opportunity to test implicit assumptions about the hierarchy of research designs (8). If particular associations between exposure and outcome were studied in both randomized, controlled trials and cohort or case-controlled studies, and if these studies were then included in meta-analysis, the results could be compared according to study design, as has been done for trials with historical controls $(5,8)$.

In the past, comparative studies have repeatedly demonstrated no significant difference between randomized, controlled trials or observational reports. The distinction between random sampling and randomization (random allocation) also has been misunderstood. The purpose of random sampling is to choose a group that is a representative of a larger population, and random sampling is not usually employed in controlled trials. The purpose of randomization, on the other hand, is to divide a single group into groups that differ only by chance. Randomization is the only way to ensure that individuals entered into a trial are not allocated to the treatment or control groups in a biased manner. The results of this study show there are no significant differences by various types of allocation of patients into groups, except with randomization, which demonstrated differences in only one aspect in two of three samples.

The results of this study show that patients presenting to interventional pain management setting are predominantly female, with a mean age of $47+0.8$ years, mean height of $67+0.2$ inches, mean weight of $180+2$ lbs., with duration of $102+6.4$ months, with $51 \%$ of the patients with gradual onset with $29 \%$ with history of previous surgery and $69 \%$ referred by a physician.

Ninety-three percent of the patients showed involvement of spine, whereas only $7 \%$ of the patients presented with non-spinal pain. Fifty six percent of the patients presented with involvement of at least two regions, whereas, $22 \%$ of the patients presented with involvement of three or more regions. Involvement of cervical spine and lumbar spine was seen in $24 \%$, whereas cervical spine, thoracic spine and lumbar spine was involved in $11 \%$ of the patients.

Fifty-eight percent of the patients presented with depression, compared to $54 \%$ with generalized anxiety disorder and $34 \%$ with somatization disorder. Nonphysiologic symptoms and signs were seen each in $15 \%$ of the patients, whereas symptom magnification was seen in $24 \%$ of the patients.

Seventy-four percent of the patients were treated with interventional procedures compared to $19 \%$ of the patients treated without interventional procedures. Seven percent of the patients underwent evaluation only. The dropout rate was $25 \%$. Reasons for dropout were multiple, including lack of insurance approval, lack of response, drug abuse and other miscellaneous reasons.

The results of this study show that there was only one significant difference among the groups when they were allocated by multiple means, without randomization (Table 1). Randomization also showed similarity among the groups, however; significant, differences were noted, in seven aspects (Table 4). The proponents of randomization agree that these differences may be considered the result of chance. However, the results show that allocation by other means may be superior to randomization thus avoiding numerous difficulties. This is a preliminary study demonstrating allocation to be reliable by any means as long as the physician is not involved in influencing the results. Thus, allocation by multiple means, either by serial numbers, by date of admission, by randomization into equal groups, or by random sampling into small groups, is equally effective.

Evidence-based medicine is a loose term, which has been used based not only on the necessity to present a particular view, but also based on personal philosophy, bias and conjecture. A current definition of evidence-based medicine is: the conscientious, explicit and judicious use of current best evidence in making decisions about the care of individual patients. Thus, evidence-based medicine is essentially what most clinicians have been trying to practice all their working lives. The practice of evidencebased medicine requires the integration of individual clinical expertise with the best available external clinical evidence from systematic research. Thus, apart from the results of randomized, controlled trials, there are a multitude of other evidence-based sources for clinical and policy decisions. All valid, relevant evidence should be considered in the decision-making process. Thus, no one sort of evidence should necessarily be the determining factor in decision-making (9). "All" implies that there should be an active search for all that is valid, relevant information and that an assessment should be made of the accuracy of information and the applicability of the evidence to the decision in question (10). Straus et al (11) described many misperceptions of evidence-based medicine. They state that rather than denigrating clinical 
experience $(12,13)$, evidence-based medicine acknowledges expertise as the basis for all clinical practice. They also state that evidence-based medicine promotes patient values and clinician independence. It has also been suggested that the criticism of evidence-based medicine as an ivory-tower concept (14) which is not the case (1527). Strauss et al (11) observed that it is a misperception that only randomized, controlled trials or systematic reviews constitute evidence-based medicine $(14,28)$. Thus, evidence-based medicine emphasizes the consideration of evidence from various types of studies appropriate to different clinical questions. The questions have been raised about the validity of evidence-based medicine when observation reports, and even unpublished observations have been excluded from consideration in a systematic review or guideline development, etc.

In spite of overwhelming evidence that evidence-based medicine includes all types of evidence, only the randomized, double-blind studiesare considered to represent the best available evidence. Randomized, double-blind trials have been considered the gold standard in judging the effectiveness of therapy. Many stumbling blocks, including the issues of ethics, feasibility, cost and reliability, pose frequently insurmountable challenges to randomized, double-blind trials in interventional pain management (29-35). Further, the value of the so-called gold standard of randomized, double-blind trials has been questioned. Benson and Hartz (36) outlined several advantages of observational studies over randomized, controlled trials, including lower costs, greater timeliness and a broader range of patients. Concato et al (8) in evaluating various types of clinical evaluations concluded that average results of observational studies were remarkably similar to those of randomized, controlled trials and that the results of well-designed observational studies do not systematically overestimate the magnitude of the effects of treatment as compared with those in randomized, controlled trials on the same topic.

In spite of a strong argument for other types of evidence, clinical research worships at the shrine of the randomized, controlled trial. The ability to assign subjects randomly to either experimental or controlled status is considered as science that is unsurpassed. However, random assignment does not confer an absolute protection against bias. It simply reduces the likelihood that such bias has occurred. Because randomized, controlled trials are complicated and difficult to conduct, they are usually restricted to very tightly targeted groups of patients. Often, the investigators are not actively concerned about how the sub- jects are obtained and rely on random allocation to distribute any differences equally across the two groups. As a result, randomized trials often trade internal validity (tightness of comparisons) for external validity (generalizability) (37). Hence, randomization does not provide the protective shield that some think. Further, many patients refuse to participate in the process with the belief that randomization always puts them in the control groups. Thus, it does not seem feasible to rely exclusively on randomized, controlled trials for all, or even most, of the needed empirical data linking outcomes to the process of care (8). Generally, a difference in outcome between a treatment and a control group can be due to chance, confounding, or bias due to differences between the groups, differences in handling the groups; and the true effect of intervention. Confounding and bias are avoided in the design of a trial by randomization, single-blinding or double-blinding. Thus, randomization is considered as a cornerstone to avoid bias and to maintain similarity between treatment and control groups, thus influencing the eventual outcome. Randomization by the tossing of a coin (or any equivalent method) ensures that the physician running the trial is not consciously or unconsciously allocating the certain patients to a particular group. Without randomization, trials of surgical versus medical technique are wide open to selection bias. It is assumed that low-risk cases are much more likely to be assigned to the operative group, leaving high-risk patients to be managed by the physicians. Assigning volunteers to the treatment group and those who do not volunteer to the control group is also likely to result in a biased comparison-volunteers will be quite different, in many respects, from patients who do not volunteer (38). The criticism also has been advanced against allocation to treatment or control groups based on alternate days, alternate numbers or another assigned preformed methodology. Even though, it is believed that randomization does ensure that the two groups will differ only by chance, it does not guarantee that in practice, the balance will be actually achieved through the randomization.

\section{CONCLUSION}

The results of this study show that in an interventional pain management setting, $29 \%$ of the patients have undergone previous surgery, $49 \%$ of the patients developed pain problems following an incident, female patients constituted $60 \%$, spinal pain constituted $93 \%$ and $56 \%$ of the patients suffered with more than one region involvement. Sixty percent of the patients presenting to this interventional pain management practice presented 
with one or a combination of multiple psychological disorders including depression, generalized anxiety disorder and somatization. Interventional procedures were performed in $74 \%$ of the patients with a total dropout rate of $25 \%$ from the program.

Comparison of the samples allocated by two nonrandomized means, showed basically no significant differences except by chance which was demonstrated only in one parameter. However, randomization showed significant differences, in one to two parameters, in each mode of randomization or random sampling. Thus, we conclude that any systematic type of allocation will yield valid results with similar groups of patients with or without randomization. However, further studies into the validity of this concept needs to be evaluated.

\section{ACKNOWLEDGMENTS}

We would like to thank Carla D. Beyer, RN, Kim S. Damron, RN, Renee C. Barnhill, RN, Linda Smith, RN, and Valeria Douglas, RN; Marla K. Neihoff and Lorie A. Caldwell, medical records coordinators; and Tonie D. Hatton, transcriptionist, for their assistance in the completion of the study and transcription of the manuscript.

\section{REFERENCES}

1. Streptomycin treatment of pulmonary tuberculosis: A Medical Research Council investigation. BMJ 1948; 2:769-782.

2. Byar DP, Simon RM, Friedewald WT et al. Randomized clinical trials: Perspectives on some recent ideas. N Engl J Med 1976; 295:74-80.

3. Feinstein AR. Current problems and future challenges in randomized clinical trials. Circulation 1984; 70:767-774.

4. Abel U, Koch A. The role of randomization in clinical studies: Myths and beliefs. J Clin Epidemiol 1999; 52:487-497.

5. Sacks H, Chalmers TC, Smith H Jr. Randomized versus historical controls for clinical trials. Am J Med 1982; 72:233-240.

6. Evidence-Based Medicine Working Group. Evidencebased medicine: A new approach to teaching the practice of medicine. JAMA 1992; 268:2420-2425.

7. Preventive Services Task Force. Guide to clinical preventive services: Report of the US Preventive Services Task Force. Second edition. Williams \& Wilkins, Baltimore, 1996.

8. Concato J, Shah N, Horwitz RI. Randomized, controlled trials, observational studies, and the hierarchy of research designs. N Engl J Med 2000; 342:1887-1892.

9. Manchikanti L. Evidence-based interventional pain medicine: Is there any evidence? Pain Physician 2002; 5:1-7.

10. McQuay H, Moore A (eds). An evidence-based resource for pain relief. Oxford University Press, New York, 1998.

11. Straus S, McAlister F, Cook D et al. Expanded philosophy of evidence-based medicine. In Guyatt G, Rennie D (eds). Users' Guides to the Medical Literature. AMA Press, Chicago, 2002, pp 211-222.

12. Charlton BG. Restoring the balance: Evidence-based medicine put in its place. J Eval Clin Pract 1997; 3:87-98.

13. Sackett DL, Straus SE. Finding and applying evidence during clinical rounds. The "evidence cart." JAMA 1998; 280:1336-1338.

14. Hampton JR. Evidence-based medicine, practice variations and clinical freedom. J Eval Clin Pract 1997; 3:123-131.

15. McColl A, Smith H, White P et al. General practitioners' perceptions of the route to evidence based medicine: A questionnaire survey. BMJ 1998; 316:361-365.

16. Tunis SR, Hayward RS, Wilson MC et al. Internists' attitudes about clinical practice guidelines. Ann Intern Med 1994; 120:956-963.

17. McAlister FA, Graham I, Karr GW et al. Evidencebased medicine and the practicing clinician. J Gen Intern Med 1999; 14:236-242.

18. Hagdrup N, Falshaw M, Gray RW et al. All members of primary care team are aware of importance of evidence based medicine. BMJ1998; 317:282.

19. Ghali WA, Saitz R, Eskew AH et al. Evidence-based medicine: Behaviors, skills, and attitudes of medical students. Ann RCPSC 1998; 31:177-182.

20. Olatunbosun OA, Edouard L, Pierson RA. Physicians' attitudes toward evidence based obstetric practice: A questionnaire survey. BMJ 1998; 316:365-366.

21. Mayer J, Piterman L. The attitudes of Australian GPs to evidence-based medicine; A focus group study. Fam Pract 1999; 16:627-632.

22. Ellis J, Mulligan I, Rowe J et al. Inpatient general medicine is evidence based. Lancet 1995; 346:407410.

23. Geddes JR, Game D, Jenkins NE et al. What proportion of primary psychiatric interventions are based on randomized evidence? Qual Health Care 1996; 5:215-217.

24. Gill P, Dowell AC, Neal RD et al. Evidence based general practice: A retrospective study of interventions in our training practice. BMJ 1996; 312:819-821.

25. Kenny SE, Shankar KR, Rintala R et al. Evidence- 
based surgery: Interventions in a regional pediatric surgical unit. Arch Dis Child 1997; 76:50-53.

26. Baraldini V, Spitz L, Pierro A. Evidence-based operation in pediatric surgery. Pediatr Surg Int 1998; 13:331-335.

27. Howes N, Chagla L, Thorpe M et al. Surgical practice is evidence based. Br J Surg 1997; 84:1220-1223.

28. Swales JD. Evidence-based medicine and hypertension. J Hypertens 1999; 17:1511-1516.

29. Hopwood MB, Manning DC. Lumbar epidural steroid injections: Is a clinical trial necessary or appropriate? Reg Anesth Pain Med 1999; 24:5-7.

30. Turner JA, Loeser JD, Bell KG. Spinal cord stimulation for chronic low back pain. A systematic literature synthesis. Neurosurgery 1995; 37:10881098.

31. Weinstein JN. The tortoise and the hare. Is there a place in spine surgery for randomized trials? Spine 1999; 23:2548-2549.

32. Winter RB. The prospective, randomized, controlled clinical trial in spine surgery. Fact or fiction? Spine
1999; 23:2550-2552.

33. Fairbank J. Randomized controlled trials in the surgical management of spinal problems. Spine 1999; 23:2556-2563

34. Tosteson TD. Point of view. Spine 1999; 24:25622563.

35. Carey TS. Randomized controlled trials in surgery. An essential component of scientific progress. Spine 1999; 23:2553-2555.

36. Benson K, Hartz AJ. A comparison of observational studies and randomized, controlled trials. $N$ Engl $J$ Med 2000; 342:1878-1886.

37. Kane RL. Approaching the outcomes question. In Kane RL (ed). Understanding Health Care Outcomes Research. Aspen Publication, Gaithersburg, 1997, pp $1-15$.

38. Daly LE, Bourke GJ. Epidemiological and clinical research methods. In Daly LE, Bourke GJ (eds). Interpretation and Uses of Medical Statistics. Blackwell Science, 2000, Oxford, pp 143-201. 